\title{
Influência da temperatura de infiltração de alumínio gasoso em ligninocelulósicos nas propriedades de $\mathrm{Al}_{2} \mathrm{O}_{3}$ biomórfica
}

\section{(Effect of the temperature of aluminum gas infiltration into lignocellulosic preforms on the properties of biomorphic $\mathrm{Al}_{2} \mathrm{O}_{3}$ )}

\author{
T. E. Andrade Jr. ${ }^{1}$, C. R. Rambo ${ }^{2}$, H. Sieber ${ }^{3}$, A. E. Martinelli ${ }^{1}$, D. M. A. Melo ${ }^{1}$ \\ ${ }^{1}$ Universidade Federal do Rio Grande do Norte, CCET-UFRN, Campus Lagoa Nova s/n, Natal, RN 59072-970 \\ ${ }^{2}$ Departamento de Engenharia Química EQA/UFSC, Campus Universitário s/n, C.P. 476, Trindade, \\ Florianópolis, SC 88040-900 \\ ${ }^{3}$ University of Erlangen-Nuremberg, Department of Materials Science, Glass and Ceramics, \\ Martensstrasse 5, D-91058 Erlangen, Alemanha \\ aemart@uol.com.br
}

\begin{abstract}
Resumo
A conversão de materiais ligninocelulósicos em cerâmicas biomórficas tem despertado particular interesse, por tratar-se de uma nova classe de materiais cerâmicos de alto valor agregado, produzidos a partir de matérias-primas de baixo custo e relevante papel econômico. Cerâmicas biomórficas são obtidas por meio do processo de biomodelagem, que consiste na reprodução ao nível micrométrico da estrutura natural do material de partida. Neste trabalho, os materiais ligninocelulósicos utilizados como material de partida foram fibras de sisal, ratam e pinheiro. Estes foram convertidos por pirólise em pré-formas de carbono. Subseqüentemente, essas pré-formas foram infiltradas com alumínio gasoso em temperaturas que variaram de $1400{ }^{\circ} \mathrm{C} \mathrm{a} 1600{ }^{\circ} \mathrm{C}$ sob vácuo, obtendo-se assim carbeto de alumínio $\left(\mathrm{Al}_{4} \mathrm{C}_{3}\right)$. Por ser um material frágil e de rápida decomposição, $\mathrm{O} \mathrm{Al}_{4} \mathrm{C}_{3}$ foi rapidamente oxidado a 1600 ${ }^{\circ} \mathrm{C}$ para sua conversão total em fibras de $\mathrm{Al}_{2} \mathrm{O}_{3}$ biomórfica. A microestrutura e morfologia das cerâmicas biomórficas obtidas foram caracterizadas por microscopia eletrônica de varredura e por difração de raios X. O estudo comparativo da infiltração de alumínio gasoso foi realizado com base em resultados obtidos por análise termogravimétrica.
\end{abstract}

Palavras-chave: ligninocelulósicos, biomodelagem, cerâmicas biomórficas.

Abstract

The reproduction of lignocellulosic structures into biomorphous ceramics has been extensively studied since it results in addedvalue products from relatively inexpensive raw materials. Synthetic ceramics can be obtained by biotemplating maintaining the structural features of native wood or fibers. In the present study, lignocellulosic materials, i.e, sisal, rattan and pinnus, were used to produce alumina fibers. The natural structures were pyrolized into carbon prefoms and subsequently infiltrated with aluminum gas temperatures ranging from $1400{ }^{\circ} \mathrm{C}$ to $1600{ }^{\circ} \mathrm{C}$ under vacuum, resulting in aluminum carbide $\left(\mathrm{Al}_{4} \mathrm{C}_{3}\right)$. Because of its brittle nature and fast decomposition, $\mathrm{Al}_{4} \mathrm{C}_{3}$ was quickly oxidized at $1600^{\circ} \mathrm{C}$ to produce biomorphous $\mathrm{Al}_{2} \mathrm{O}_{3}$ fibers. The microstructure and morphology of the ceramic fibers was characterized by scanning electron microscopy and X-ray diffraction. A comparative study on the infiltration of aluminum gas was performed based on thermogravimetric analyses.

Keywords: lignocellulosic materials, biotemplating, biomorphous ceramics.

\section{INTRODUÇÃO}

O desenvolvimento de cerâmicas biomórficas tem aumentado nos últimos anos devido à possibilidade de se reproduzir macro e microporos que não poderiam ser reproduzidos por técnicas convencionais. Desta forma, obtêm-se cerâmicas com propriedades específicas que incluem alta área superficial, alta permeabilidade, estabilidade em altas temperaturas, baixo peso específico e baixa condutividade térmica. Tais materiais têm sido investigados para uma variedade de aplicações, tais como filtros, componentes estruturais de baixa densidade, eletrodos, sensores, bioreatores e catalisadores $[1,2]$.

A conversão de estruturas bioorgânicas em cerâmicas biomórficas é alcançada por meio de biomodelagem. Esta metodologia recentemente desenvolvida consiste basicamente na conversão, em escala micrométrica, de bioestruturas em materiais cerâmicos. Após o processamento, cerâmicas biomórficas exibem morfologia de fibras ou poros unidirecionais (estrutura celular), similares a do material de partida. Essa técnica oferece a possibilidade de se produzir uma ampla variedade de materiais cerâmicos como carbetos, nitretos e óxidos, entre outros, com diferentes propriedades e, consequentemente, viáveis para diferentes aplicações [3, 4]. 
Exemplos de biomodelagem, chamados de biomineralização, são freqüentemente encontrados na própria natureza [5]. A fossilização é um processo conhecido, no qual a estrutura de um material orgânico é convertida em um mineral natural. Este processo, no entanto, leva milhares de anos e não se mostra viável para uso prático em larga escala na engenharia de materiais. Buscam-se, então, novos métodos de biomodelagem, com baixo custo de processamento [6].

Trabalhos anteriores de biomodelagem foram realizados visando à obtenção de cerâmicas biomórficas de carbeto de silício partindo-se de estruturas ligninocelulósicas, através de diferentes rotas de processamento, tais como: infiltração de silício no estado líquido em madeira pirolizada [7], infiltração de gases de $\mathrm{SiO}$ e $\mathrm{Si}$ [8] e infiltrações com polímeros e monômeros de silício [9]. Um aumento significante na velocidade de transformação pode ser obtido quando as bioestruturas de partida com porosidade aberta, acessíveis a infiltrantes líquidos e gasosos, são submetidas a processos reativos a altas temperaturas, geralmente entre $1200^{\circ} \mathrm{C}$ e $1700^{\circ} \mathrm{C}[10]$.

O método de obtenção de cerâmicas biomórficas a partir de estruturas naturais, ou seja, através de materiais ligninocelulósicos, pode ser baseado na infiltração de gás de alumínio na microestrutura do carbono, obtida por pirólise de um material natural ligninocelulósico, resultando em $\mathrm{Al}_{4} \mathrm{C}_{3}$ [11]. Normalmente, o carbeto de alumínio é uma fase cerâmica não desejada, devido à falta de estabilidade química em ambientes úmidos, transformando-se rapidamente em hidróxido de alumínio que se expande com o passar do tempo, afetando significativamente as propriedades mecânicas do sistema [12]. Devido à falta de estabilidade do material, é necessária uma posterior oxidação para obtenção de $\mathrm{Al}_{2} \mathrm{O}_{3}[11]$.

\section{MATERIAIS E MÉTODOS}

A obtenção de $\mathrm{Al}_{2} \mathrm{O}_{3}$ biomórfica foi realizada a partir de materiais ligninocelulósicos, fibras de sisal, ratam e pinheiro. As fibras de sisal foram cortadas com $10 \mathrm{~cm}$ de comprimento. As amostras de ratam e pinheiro foram cortadas em discos de aproximadamente $2,0 \mathrm{~cm}$ (ratam) e 1,5 (pinheiro) de diâmetro e $1,0 \mathrm{~cm}$ de altura e secados a $130{ }^{\circ} \mathrm{C}$ por $2 \mathrm{~h}$. Após a secagem, as amostras foram pirolisadas a $800{ }^{\circ} \mathrm{C}$ por $1 \mathrm{~h}$ em atmosfera de nitrogênio para decomposição dos biopolímeros (celulose, hemicelulose e lignina). Durante a pirólise, a taxa de aquecimento utilizada foi baixa $\left(1,5^{\circ} \mathrm{C} /\right.$ $\mathrm{min}$ ) para impedir danos às paredes celulares, eventualmente causados pela rápida liberação de gases.

Após a etapa de pirólise, as amostras foram submetidas ao processo de infiltração com vapor de alumínio por $1 \mathrm{~h}$. Durante a infiltração em vácuo (1-0.1 Pa) a temperaturas na faixa de $1400{ }^{\circ} \mathrm{C}$ a $1600{ }^{\circ} \mathrm{C}$, o bio-carbono $\left(\mathrm{C}_{\mathrm{B}}\right)$ reage com vapor de $\mathrm{Al}$, formando $\mathrm{Al}_{4} \mathrm{C}_{3}$. Após um processo de oxidação/ sinterização a $1600{ }^{\circ} \mathrm{C}$ por $3 \mathrm{~h}$, a uma razão de aquecimento de $10{ }^{\circ} \mathrm{C} / \mathrm{min}$ sem atmosfera circulante, os preformados de $\mathrm{Al}_{4} \mathrm{C}_{3}$ foram convertidos em $\mathrm{Al}_{2} \mathrm{O}_{3}$. Essa metodologia é descrita em detalhes em publicação recente [11]. As etapas de obtenção do material utilizado neste trabalho foram realizadas em forno horizontal.

Os materiais obtidos durante o processo foram caracterizados por microscopia eletrônica de varredura utilizando-se um equipamento Philips XL-30 e por difratometria de raios $X\left(10^{\circ} \leq 2 \theta \leq 80^{\circ}\right)$, utilizando um equipamento Siemens modelo D5000 e radiação $\mathrm{CuK \alpha}$. $\mathrm{O}$ estudo comparativo das temperaturas de infiltrações do alumínio gasoso foi realizado através de análise termogravimétrica de perda de massa em função da temperatura de infiltração do vapor de $\mathrm{Al}$, realizados em uma termobalança Perkin-Elmer modelo TGA-7. As análises foram realizadas desde a temperatura ambiente até $800{ }^{\circ} \mathrm{C}$ para se determinar a quantidade de carbono não reativo após o processo. Acima de $900{ }^{\circ} \mathrm{C}$, o carbeto de alumínio oxida-se e forma alumina, resultando em ganho de massa. A densidade da fração sólida dos materiais foi obtida por picnometria ao hélio. A porosidade das amostras foi calculada pela relação entre as densidades geométrica $\left(\rho_{\mathrm{G}}\right)$ e picnométrica $\left(\rho_{\mathrm{p}}\right)$.

\section{RESULTADOS E DISCUSSÃO}

As amostras de carbeto de alumínio das fibras de sisal, ratam e pinheiro foram oxidadas a $1600{ }^{\circ} \mathrm{C}$ e convertidas em $\alpha$-alumina, conforme ilustra o difratograma de raios $\mathrm{X}$ da Fig. 1, obtido após conversão do sisal.

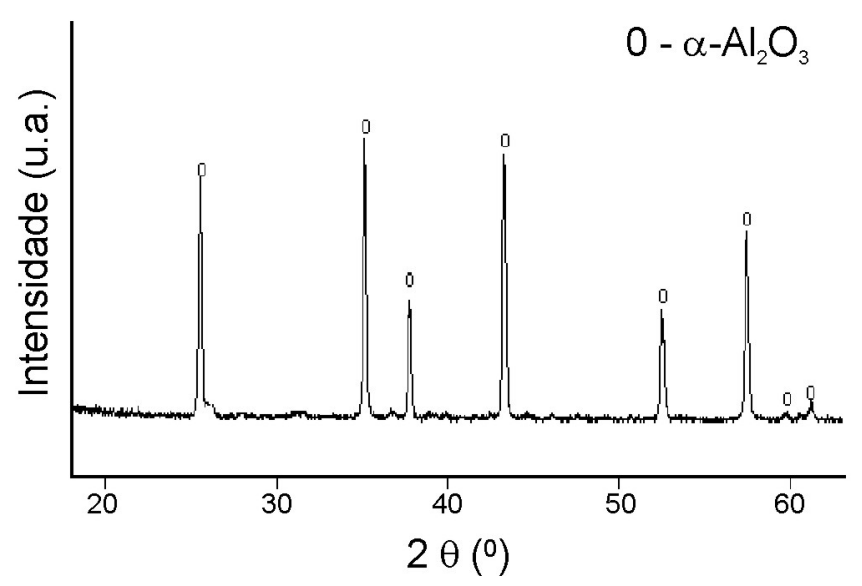

Figura 1: Difração de raios $\mathrm{X}$ de alumina biomórfica derivada de fibras de sisal.

[Figure 1: X-ray diffraction pattern of biomorphic alumina produced from sisal.]

A Fig. 2 apresenta a morfologia interna da fibra de sisal (A), da fibra de sisal pirolisada (B) e a da alumina biomórfica derivada do sisal (C). A anatomia das fibras de sisal é caracterizada por uma composição multicelular de pequenas células, longitudinalmente dispostas e unidas. Depois de pirolisada, a anatomia das fibras foi mantida e a morfologia das fibras pode ser claramente identificada. Os vasos condutores podem ser observados nas Figs. 2B e 2C e podem ser caracterizados por uma morfologia poligonal com lúmen arredondado. As células estão mais abertas 

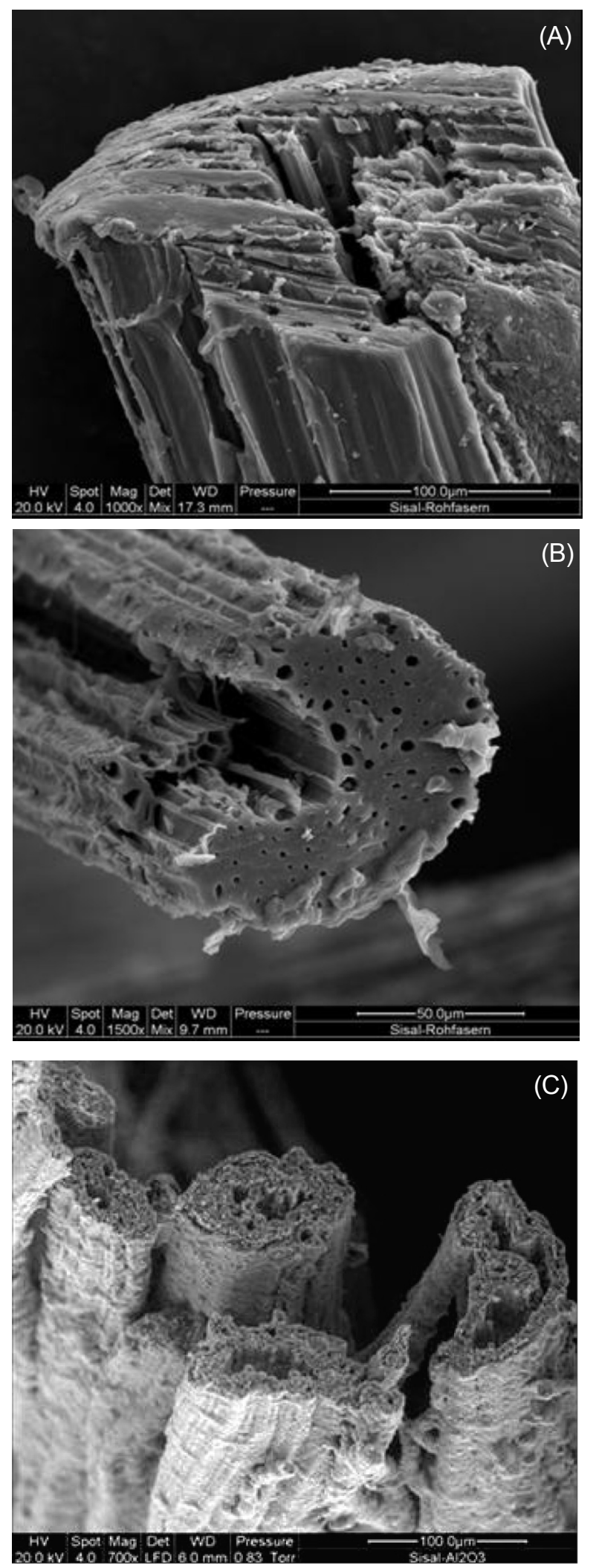

Figura 2: Microscopia eletrônica de varredura da morfologia interna, da fibra de sisal (A), da fibra de sisal pirolisada (B) e a da alumina biomórfica derivada do sisal.

[Figure 2: Scanning electron microscopy images of internal morphology of sisal fiber (A), pyrolized sisal fiber (B) and biomorphic alumina produced from sisal.]

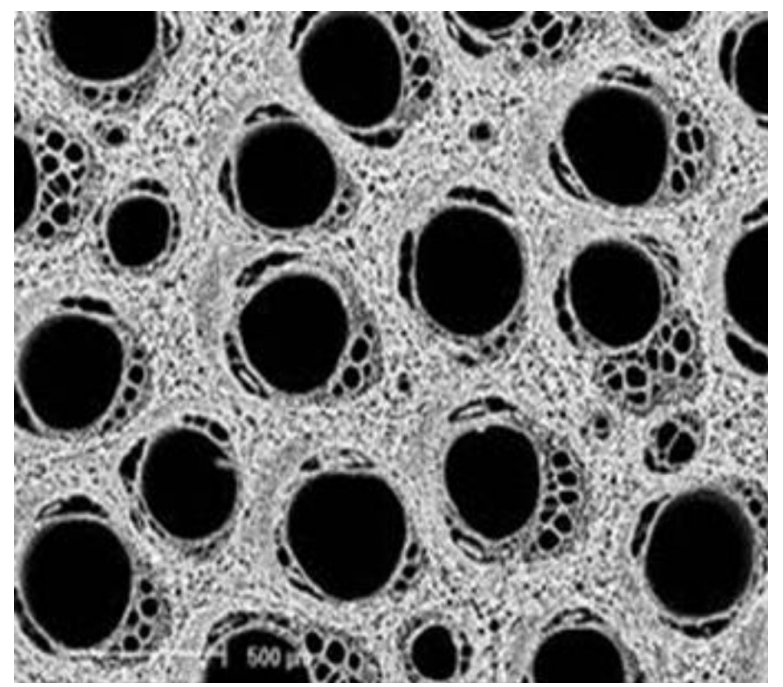

Figura 3: Microscopia eletrônica de varredura do ratam pirolisada. [Figure 3: Scanning electron microscopy image of pyrolized rattan.]
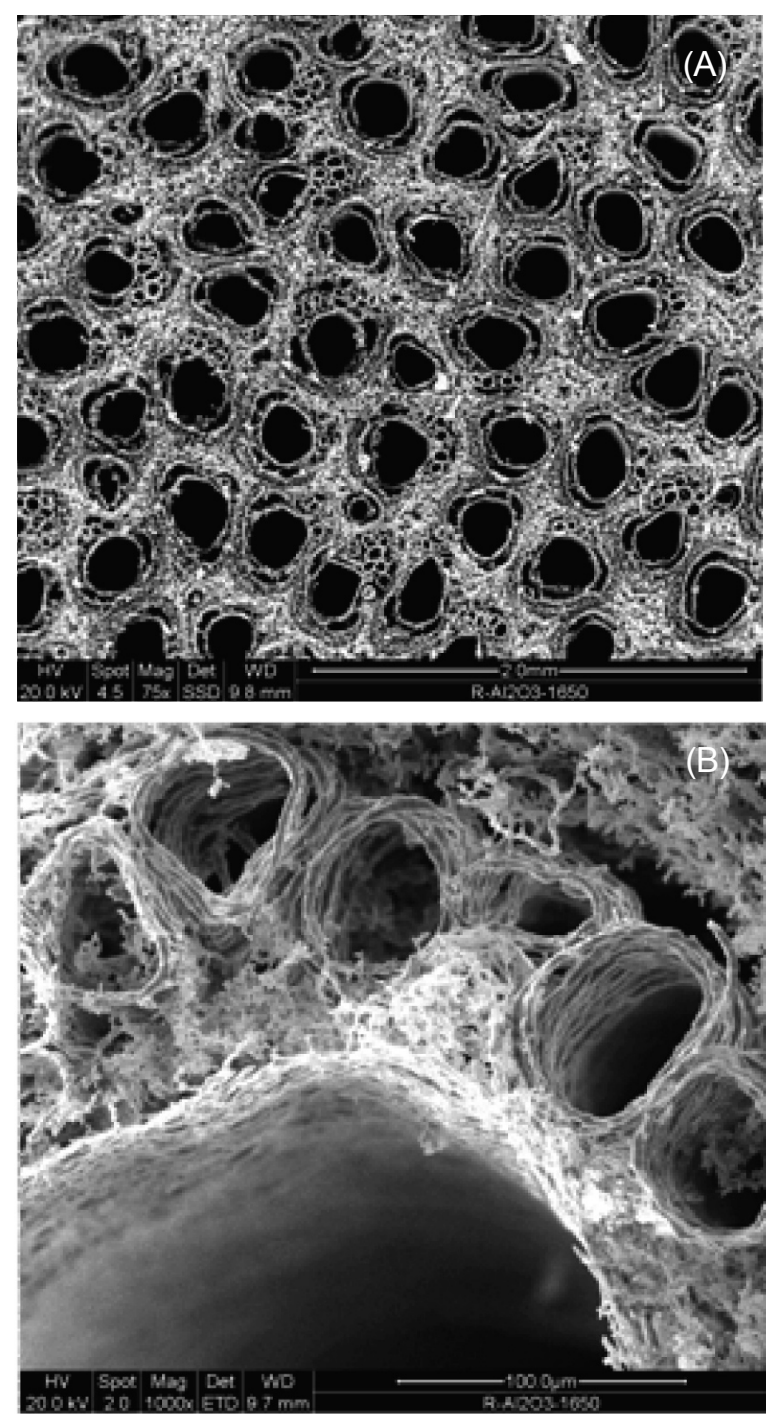

Figura 4: Microscopia eletrônica de varredura da alumina biomórfica derivada do ratam.

[Figure 4: Scanning electron microscopy images of biomorphic alumina produced from rattan.] 


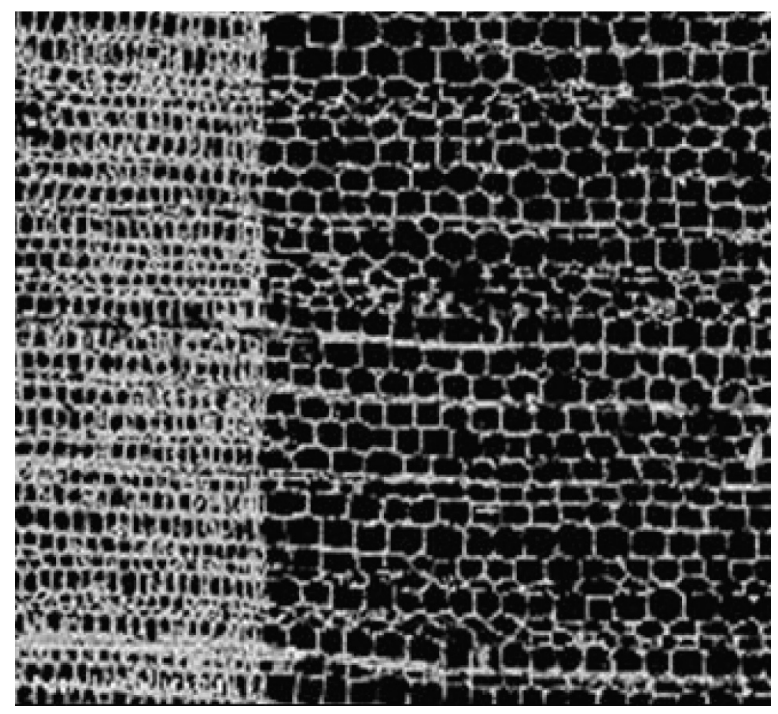

Figura 5: Microscopia eletrônica de varredura do pinheiro pirolisado.

[Figure 5: Scanning electron microscopy image of pyrolized pinus.]

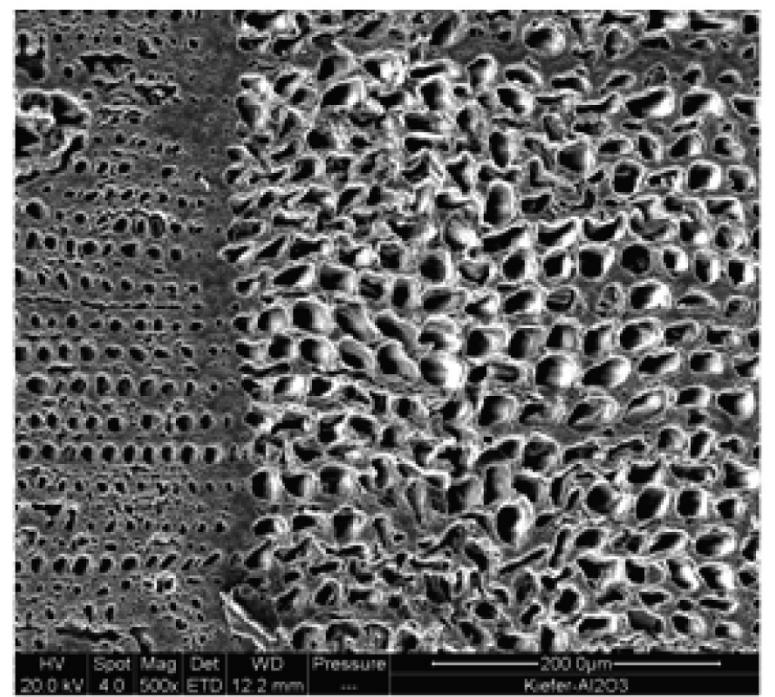

Figura 6: Microscopia eletrônica de varredura da alumina biomórfica derivada do pinheiro.

[Figure 6: Scanning electron microscopy image of biomorphic alumina produced from pinus.]

devido à perda de massa orgânica durante a pirólise (Fig. 2B), facilitando a infiltração de alumínio gasoso e obtenção de $\mathrm{Al}_{2} \mathrm{O}_{3}$ biomórfica com morfologia semelhante ao do material de partida.

As Figs. 3 e 4 apresentam, respectivamente, micrografias do ratam pirolisado e da alumina biomórfica derivada do ratam, obtida após infiltração com alumínio gasoso e posterior oxidação. Na Fig. 3, pode-se observar grandes vasos $(200-300 \mu \mathrm{m})$ característicos do meta-xilema, como também vasos de tamanho médio (aproximadamente $90 \mu \mathrm{m}$ ), característicos do floema. A Fig. 4 apresenta micrografias da alumina biomórfica derivada do ratam (cortes axiais).
Todas as características morfológicas do ratam pirolisado foram mantidas após oxidação do $\mathrm{Al}_{4} \mathrm{C}_{3} \mathrm{em} \mathrm{Al}_{2} \mathrm{O}_{3}$. Na Fig. $4 \mathrm{~A}$, pode ser observada a reprodução de grandes vasos (200$330 \mu \mathrm{m})$ característicos do meta-xilema. Na Fig. 4B nota-se a reprodução de vasos médios (aproximadamente $90 \mu \mathrm{m}$ ) característicos do floema.

O pinheiro apresenta anéis sazonais (Fig. 5) caracterizados por uma região (à esquerda) de células do lenho tardio e outra (à direita) de lenho inicial.

A Fig. 6 apresenta micrografias da alumina biomórfica derivada do pinheiro (cortes axiais). Todas as características morfológicas do pinheiro pirolisado foram mantidas após oxidação do $\mathrm{Al}_{4} \mathrm{C}_{3}$ em $\mathrm{Al}_{2} \mathrm{O}_{3}$. Na Fig. 6a observa-se a reprodução dos anéis sazonais e na Fig. $6 \mathrm{~b}$ pode-se observar, claramente, a transição das células do lenho inicial (lado direito), para as células do lenho tardio (lado esquerdo).

As fibras de $\mathrm{Al}_{4} \mathrm{C}_{3}$ derivadas das fibras de sisal e infiltradas

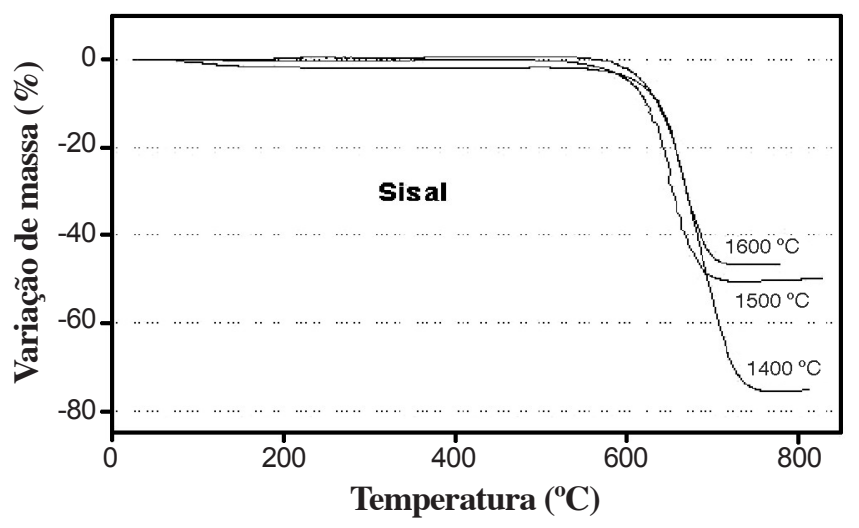

Figura 7: Análise termogravimétrica das fibras de carbeto de alumínio obtidas após infiltração com alumínio gasoso em diferentes temperaturas.

[Figure 7: Thermogravimetric analysis of aluminum carbide fibers obtained by infiltration of aluminum gas at different temperatures.]

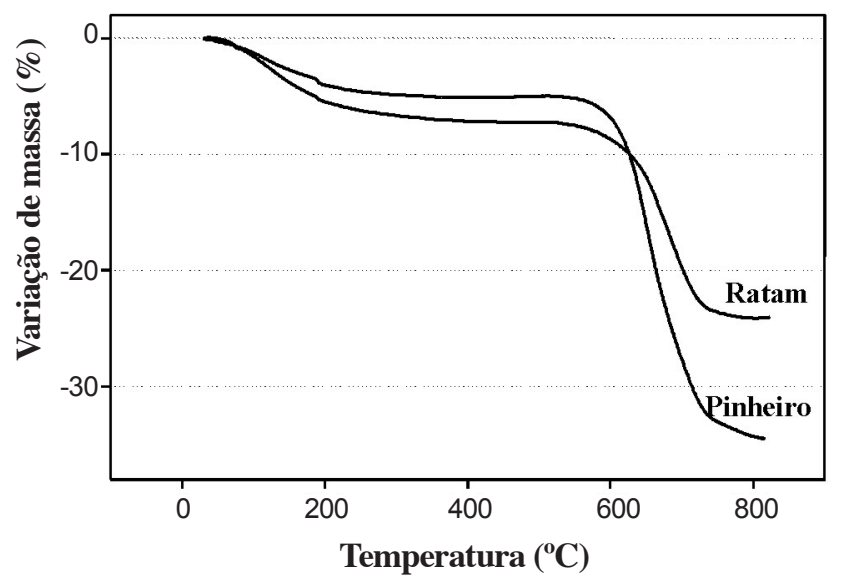

Figura 8: Análise termogravimétrica do carbeto de alumínio biomórfico derivado do ratam e pinheiro durante a oxidação.

[Figure 8: Thermogravimetric analysis of biomorphic aluminum carbide produced from rattan and pinus during oxidation.] 
com alumínio gasoso a $1400{ }^{\circ} \mathrm{C}, 1500{ }^{\circ} \mathrm{C}$ e $1600{ }^{\circ} \mathrm{C}$, foram submetidas à análise termogravimétrica ao ar, com razão de aquecimento de $10{ }^{\circ} \mathrm{C} / \mathrm{min}$, convertendo-as assim em $\mathrm{Al}_{2} \mathrm{O}_{3}$. A oxidação iniciou-se em torno de $590^{\circ} \mathrm{C}$ e finalizou a aproximadamente $700^{\circ} \mathrm{C}$. Acima de $750{ }^{\circ} \mathrm{C}$, o fim da perda de massa foi observada para todas as amostras, conforme mostra a Fig. 7. A perda de massa apresentada pelas fibras infiltradas a $1400{ }^{\circ} \mathrm{C}$ foi de aproximadamente $75 \%$, e para as fibras infiltradas entre $1500{ }^{\circ} \mathrm{C}$ e $1600{ }^{\circ} \mathrm{C}$ foi de aproximadamente $50 \%$ e $45 \%$, respectivamente. A perda de massa corresponde ao carbono que não reagiu na infiltração com alumínio gasoso e que, em atmosfera oxidante, é liberado em forma de $\mathrm{CO} / \mathrm{CO}_{2}$. Comprovase por meio dos dados obtidos, que a melhor temperatura para infiltração de alumínio gasoso nas fibras derivadas do sisal foi $1600{ }^{\circ} \mathrm{C}$.

A Fig. 8 mostra a curva termogravimétrica do carbeto de alumínio biomórfico derivado do ratam e pinheiro durante a oxidação ao ar. As perdas de massas do carbeto de alumínio foram de $23 \%$ e $35 \%$, respectivamente. Essa perda de massa corresponde à liberação de carbono restante que não reagiu na infiltração com alumínio gasoso e é liberado na forma de $\mathrm{CO} / \mathrm{CO}_{2}$.

A Tabela I mostra a densidade $\left(\rho_{\mathrm{G}}\right)$ e porosidade dos preformados de $C_{B}\left(\rho_{P}=1,5 \mathrm{~g} / \mathrm{cm}^{3}\right)$ e respectivas aluminas biomórficas $\left(\rho_{\mathrm{P}}=3,95 \mathrm{~g} / \mathrm{cm}^{3}\right)$. Em contraste com o pinheiro, os grandes poros do ratam definem sua porosidade total. Após a infiltração de Al gasoso, as células pequenas do ratam são obstruídas, aumentando a densidade do preformado [11]. O tamanho de poro do ratam aumentou, devido à liberação de carbono restante (não-reagido em $\mathrm{Al}_{4} \mathrm{C}_{3}$ ) durante a oxidação, levando ao aumento da porosidade total.

Tabela I - Propriedades físicas dos preformados de carbono $\left(\mathrm{C}_{\mathrm{B}}\right)$ e respectivas $\mathrm{Al}_{2} \mathrm{O}_{3}$ biomórficas.

[Table I - Physical properties of carbon prefoms $\left(C_{B}\right)$ and corresponding biomorphic $\left.\left.\mathrm{Al}_{2} \mathrm{O}_{3}\right)\right]$

\begin{tabular}{lcccc}
\hline Propriedade & $\begin{array}{c}\mathrm{C}_{\mathrm{B}} \\
\text { Ratam }\end{array}$ & $\begin{array}{c}\mathrm{Al}_{2} \mathrm{O}_{3} \\
\text { Ratam }\end{array}$ & $\begin{array}{c}\mathrm{C}_{\mathrm{B}} \\
\text { Pinheiro }\end{array}$ & $\begin{array}{c}\mathrm{Al}_{2} \mathrm{O}_{3} \\
\text { Pinheiro }\end{array}$ \\
\hline $\begin{array}{c}\text { Densidade } \\
\left(\mathrm{g} / \mathrm{cm}^{3}\right)\end{array}$ & $0,23 \pm 0,02$ & $0,55 \pm 0,02$ & $0,34 \pm 0,02$ & $0,49 \pm 0,02$ \\
$\begin{array}{c}\text { Porosidade } \\
(\mathrm{Vol} \%)\end{array}$ & $83 \pm 1$ & $89 \pm 1$ & $76 \pm 1$ & $87 \pm 1$ \\
\hline
\end{tabular}

\section{CONCLUSÕES}

Foi possível pirolisar materiais ligninocelulósicos (fibras de sisal, ratam e pinheiro) a $800^{\circ} \mathrm{C}$ sob atmosfera de nitrogênio, obtendo-se conversão total do material de partida em fibras de carbono, mantendo-se a mesma morfologia da bioestrutura original. As pré-formas de carbono, obtidas após pirólise, foram infiltradas com alumínio gasoso. A $1600{ }^{\circ} \mathrm{C}$ obteve-se melhor infiltração comparado com temperaturas inferiores, resultando em alumina biomórfica com melhor rendimento de conversão após oxidação do carbeto de alumínio.

\section{AGRADECIMENTOS}

À Capes/DAAD e ao CNPq pela concessão de bolsa de estudos de doutorado e pelo financiamento deste trabalho de pesquisa, respectivamente. À Fundação Volkswagen pelo suporte financeiro (Contrato I/73 043) e ao CNPq pela concessão do Projeto 476917/03-4.

\section{REFERÊNCIAS}

[1] J. Cao, C. R. Rambo, H. Sieber, Preparation of porous $\mathrm{Al}_{2} \mathrm{O}_{3}$-ceramics by biotemplating of wood, J. Porous Mater. 11 (2004) 163.

[2] P. Greil, Biomorphous ceramics from lignocellulosics, J. Eur. Ceram. Soc. 21 (2001) 105.

[3] H. Sieber, C. Hoffmann, A. Kaind, P. Greil, Biomorphic cellular ceramics, Adv. Eng. Mater. 2, (2000) 105.

[4] K. J. Duchow, R. A. Grhardt, Dieletric characterization of wood and wood infiltrated with ceramic precursors, Mater. Sci. Eng. C 4, (1996) 125.

[5] S. Mann, J. Webb, R. P. Williams, Biomineralization Chemical and biochemical perspectives, VCH, Weinheim, Germany (1989).

[6] R. F. Leo, E. S. Barghoon, Silicification of wood, Botanical Museum leaflets, Harvard University 25, (1976) 1.

[7] P. Greil, T. Lifka, A. Kaindl, Biomorphic cellular silicon carbide ceramics from wood: I. Processing and microstructure, J. Eur. Ceram. Soc. 18, 14 (1998) 1961.

[8] E. Vogli, J. Mukerji, C. Hoffmann, R. Kladny, H. Sieber, P. Greil, Conversion of oak to silicon carbide ceramic by gas-phase reaction with silicon monoxide, J. Am. Ceram. Soc. 84, 6 (2001) 1236.

[9] T. Ota, H. Ichiyama, Y. Hikichi, H. Akahane, Production of ceramic wood by mimicking fossil wood, J. Korean Ceram. Soc. 7. (1997) 21.

[10] C. Zollfrank, R, Kladny, G. Motz, H. Sieber, P. Greil, Proc. $103^{\text {rd }}$ Ann. Meet. Am. Ceram. Soc., Symp. E2: Innovative Processing and Synthesis of Ceramics, Glasses and Composites, Indianapolis, EUA, (2001).

[11] C. R. Rambo, H. Sieber, Novel Synthetic Route to Biomorphic $\mathrm{Al}_{2} \mathrm{O}_{3}$ Ceramics, Adv. Mater. 17, 8, (2005) 1088.

[12] J. K. Park, J. P. Lucas, Moisture effect on $\mathrm{SiC}_{\mathrm{P}} / 6061 \mathrm{Al}$ MMC: dissolution of interfacial $\mathrm{Al}_{4} \mathrm{C}_{3}$, Scripta Mater. 37, 4 (1997) 511.

(Rec. 29/06/2006, Ac. 22/09/2006) 